\title{
Expanding Arsenal Against Diabetes Mellitus Through Nanoformulations Loaded With Glimepiride and Simvastatin: A Comparative Study
}

Narendra Kumar Pandey

Lovely Professional University

SACHIN KUMAR SINGH ( $\nabla$ singhsachin23@gmail.com )

Lovely Professional University Department of Pharmaceutical Sciences https://orcid.org/0000-00033823-6572

\section{Bimlesh Kumar}

Lovely Professional University

\section{Monica Gulati}

Lovely Professional University

\section{Sukriti Vishwas}

Lovely Professional University Department of Pharmaceutical Sciences

\section{Rubiya Khursheed}

Lovely Professional University Department of Pharmaceutical Sciences

Harish Dureja

Maharshi Dayanand University Rohtak

Dinesh Kumar Chellappan

International Medical University

Niraj Kumar Jha

Sharda University

\section{Ankur Sharma}

Sharda University

\section{Saurabh Kumar Jha}

Sharda University

Piyush Kumar Gupta

Sharda University

\section{Saurabh Gupta}

Chitkara University

\section{Gaurav Gupta}

Suresh Gyan Vihar University

\section{Parteek Prasher}

University of Petroleum and Energy Studies 


\section{Kamal Dua}

University of Technology Sydney

\section{Research Article}

Keywords: Diabetes, Nanosuspension, Solid-SNEDDS

Posted Date: November 30th, 2021

DOI: https://doi.org/10.21203/rs.3.rs-897097/v1

License: (c) (1) This work is licensed under a Creative Commons Attribution 4.0 International License. Read Full License

Version of Record: A version of this preprint was published at Environmental Science and Pollution Research on March 7th, 2022. See the published version at https://doi.org/10.1007/s11356-022-19371-z. 


\section{Abstract}

Type 2 diabetes mellitus is one of the most common and life-threatening diseases found across the globe. It occurs due to insulin resistance (IR). Major causes of IR include obesity, sedentary life style and hyperlipidemia. Glimepiride (GLM) is one of the most common oral sulphonyl ureas that is being used to treat diabetes and Simvastatin (SIM) is one of the most common statins that is used to treat hyperlipidaemia. However, both the drugs suffer from dissolution rate limited oral bioavailability. In the present study two such drugs, GLM and SIM were co-formulated into nanosuspension (NS) as well as self-nanoemulsifying drug delivery systems (L-SNEDDS). Both formulations were spray dried for solidification and evaluated for their antidiabetic potential against high fat diet and streptozotocin induced rat model. The study showed significant $(p<0.05)$ decrease in lipid/cholesterol and blood glucose levels and significant increase in antioxidant levels in the rats treated with NS and SNEDDS containing the drugs alone as well as their combination as compared to their unprocessed forms. However, the efficacy was more prominent in case of combination possibly due to dual benefits i.e., decrease in IR due to statin and control of blood glucose level. Among NS and SNEDDS, NS was found more efficacious than that of the SNEDDS possibly due to higher enhancement of oral bioavailability in case of NS.

\section{Introduction}

Diabetes mellitus (DM) is a group of metabolic diseases in which a person has high blood sugar, either because the body does not produce enough insulin (type 1), or because cells do not respond to insulin (insulin resistance) that is produced (type 2). According to the world health organization, DM is one of the largest global health emergencies of the 21st century and it is among the top 10 causes of death globally (Khursheed et al. 2019). As per International Diabetes Federation (IDF) 2017, about 425 million people worldwide were estimated to have diabetes (Cho et al. 2018, Karuranga et al. 2018). Further, diabetes also imposes a significant economic impact on countries as well as healthcare system. About USD 232 billion was spent to treat people with diabetes worldwide in 2007(Cho et al. 2018, Karuranga et al. 2018). It reached to USD 727 billion in 2017 and expected to rise up to USD 776 billion by 2045 (Cho et al. 2018). Type 2 DM (T2DM) is more common and life threatening that occurs due to insulin resistance (IR). Major causes of IR include obesity, intake of high calorie diet, sedentary lifestyle, high lipid/cholesterol level in blood as well as pancreas and dysbiosis of gut microflora that causes release of lipo-polysaccharides (LPS). Glimepiride (GLM) belongs to the sulfonylureas class of antidiabetic drugs. It reduces blood glucose level by stimulation of beta cells of pancreas for releasing insulin (Basit et al. 2012, Rajesh et al. 2018). Simvastatin (SIM) is well known for its anti-hyperlipidemic activity. SIM reduces cholesterol, very low-density lipoproteins (VLDL) and TG (Triglycerides). Whereas, it increases HDL-C (High density lipoprotein - cholesterol) (Gazzerro et al. 2012, Sharma et al. 2018). It is well known fact that reduction in body weight, lipid/cholesterol level and exercise are the main strategies that have to be practiced along with oral antidiabetics to manage T2DM. Moreover, it has been observed that cardiomyopathy and neuropathy are the common complications that is observed in patients having long history of T2DM 
(Khursheed et al. 2019, Usman et al. 2019). There are reports of fat deposition in vital organs while using GLM alone (Khursheed et al. 2019). Thus, it is anticipated that co-administration of GLM and SIM could be able to offer an effective management of diabetes, particularly in people having long history of T2DM and who are on long-term therapy using GLM. SIM and GLM are highly lipophilic and possess dissolution rate limited oral bioavailability (Rajesh et al. 2018, Sharma et al. 2018). Hence, both the drugs were coformulated into nanosuspension (NS) and self-nanoemulsifying drug delivery system (SNEDDS) and their impact on enhancing the therapeutic efficacy of GLM and SIM in treating diabetes was investigated using high fat and streptozotocin (STZ) induced rat model.

\section{Experimental \\ 2.1. Materials}

SIM and GLM were procured from Yarrow Chem, Pvt. Ltd India. Tween 80 (T80), and aerosil 200 (A-200) were purchased from Central Drug House (CDH), New Delhi, India. Labrafil M1944CS (LMCS) and Transcutol P (TP) were gifted by M/s Gattefosse, Mumbai, India. Capmul MCM (CMCM) was obtained from M/s Abitec Corp., Ohio. Triple distilled water was used throughout the study.

\subsection{Methods}

\subsubsection{Formulation of nanosuspension}

Nanosuspension was formulated by liquid antisolvent precipitation (LAP) (Mahesh et al. 2014, Pandey et al. 2020, Singh et al. 2011). The L-SNEDDS were solidified into free-flowing powder using spray drying. Spray drying technique was used to convert L-SNEDDS into free-flowing powder. In order to prepare liquid nanosuspension, SIM (1g) and GLM (0.4g) were accurately weighed and solublized in $40 \mathrm{~mL}$ of acetone as solvent. These were poured with the help of a glass burette $(50 \mathrm{~mL})$ to a glass beaker $(1000 \mathrm{~mL})$ containing distilled water $(500 \mathrm{~mL})$ that already contained SLS $(0.59 \mathrm{~g})$ and PVP K-30 $(0.45 \mathrm{~g})$. The water was used as antisolvent. The dispersion was stirred at $4000 \mathrm{rpm}$ for $4 \mathrm{~h}$ using Silverson's homogenizer (REMI, India). A clear and transparent nanosuspension was formed. This nanosuspension was dried to obtain free flowing powder using spray drier (model: SprayMate, JISL, Navi Mumbai, India). The liquid suspension was fed to the spray nozzle $\left(0.7 \mathrm{~mm}\right.$ diameter; kept at atomization pressure of $\left.4 \mathrm{~kg} / \mathrm{cm}^{2}\right)$ at a flow rate of $20 \mathrm{~mL} / \mathrm{min}$ using a peristaltic pump. The inlet and recorded outlet temperatures were $120^{\circ} \mathrm{C}$ and $55^{\circ} \mathrm{C}$, respectively. The spray dried nanosuspension powder (SP-NS) was stored in a desiccator until further use.

\subsubsection{Formulation of solid SNEDDS (S-SNEDDS)}

The isotropic mixture $(1 \mathrm{~mL})$ was prepared using $33 \%$ v/v Capmul MCM, 1.7\% v/v Labrafil M1944CS, 1.7 $\%$ v/v tween - 80 and $33 \%$ v/v transcutol - P. To this mixture GLM (5 mg) and SIM (2 mg) were added and blended for 15 minutes using vortex mixer to form a monophasic system. Further, $185 \mathrm{mg}$ of A-200 was added to convert the liquid SNEDDS into free-flowing powder. This powder was dissolved in $100 \mathrm{~mL}$ of 
ethanol and the formed dispersions were spray dried under following conditions (Ghosh et al. 2020, Khursheed et al. 2020, Kumar et al. 2018): Nozzle diameter $-0.7 \mathrm{~mm} ; 16 \mathrm{~mL} / \mathrm{min}$ feed pump flow rate; atomizer air pressure: $4 \mathrm{Kg} / \mathrm{cm}^{2} ; 70^{\circ} \mathrm{C}$ inlet and $36^{\circ} \mathrm{C}$ outlet air temperature.

\subsubsection{Characterization of prepared formulation \\ 2.2.3.1. Drug loading}

L-SNEDDS (optimized batch), SSNEDDS and SP-NS were used for the determination of drug loading. A known volume $(5 \mathrm{ml})$ was taken from L-SNEDDS and centrifuged at $11200 \mathrm{~g}$ for $15 \mathrm{~min}$ to remove undissolved drugs. Supernatant was collected and diluted appropriately using distilled water and concentration of both the drugs, GLM and SIM were estimated using RP-HPLC at $232 \mathrm{~nm}$. Drug loading (\%) was calculated using given formula (Eq. 1). The same procedure was used for S-SNEDDS and SP-NS (Garg et al. 2019, Garg et al. 2017).

\%Drugloading $=\frac{\text { AreaoftestdrugpresentinSNEDDS }}{\text { Areaofknownstandard }} \times 100$ Eq. (1)

\subsubsection{Droplet size, polydispersity index (PDI) and zeta potential}

The mean droplet size, PDI and zeta potential of developed formulations (SP-NS, L-SNEDDS and SSNEDDS) was determined using Malvern's zeta sizer (Nano ZS90, Malvern Instruments Ltd., UK). A laser beam at an angle of 90 degree with a voltage of $50 \mathrm{mV}$ was passed through the polystyrene cells containing samples. The results were recorded by conducting 12 -subruns $/ 2$ minutes. The study was carried out in triplicate and mean data was recorded (Inugala et al. 2015).

\subsubsection{Pharmacodynamics study}

The antidiabetic study was conducted on adult male Wistar rats of having age between 7 to 8 weeks and weight between 250-350 g. The rats were purchased from National Institute of Pharmaceutical Education and Research (NIPER), Mohali, India and kept in propylene lined cages having bedding of husk. The rats were stored at $25 \pm 2^{\circ} \mathrm{C}$ and $55 \pm 10 \%$ relative humidity in 12 hours light and dark cycle. Standard pellet diet and water ad libitum were used to feed rats. The experimental protocol (Protocol Number:

LPU/IAEC/2018/24) was scrutinized and got approval for ethical conduct of experiment by Institutional Animal Ethics Committee of School of Pharmaceutical Sciences, Lovely Professional University.

\subsubsection{Induction of diabetes and obesity}

All the animals were randomly divided into 17 groups (Table 1). High fat diet (HFD) was given to all the animals for 14 days after that induction of diabetes was performed using a single intraperitoneal injection of $45 \mathrm{mg} / \mathrm{kg}$ streptozotocin (STZ) in ice cold $0.1 \mathrm{M}$ sodium-citrate buffer ( $\mathrm{pH}$ 4.5). Age-matched control rats have received an equivalent amount of sodium-citrate buffer. The rats with blood glucose greater than $200 \mathrm{mg} / \mathrm{dL}$ were found as diabetic and considered for further study (Garg et al. 2019). 
Table 1

Pharmacodynamic study design ( $\mathrm{n}=6$ in each group).

\section{Groups Treatment}

I Normal control (NC)
Dose (Route of Administration)

Ice cold $0.1 \mathrm{M}$ sodium-citrate buffer (pH 4.5), p.o

\section{$45 \mathrm{mg} / \mathrm{kg}$ Streptozotocin (STZ) in ice cold $0.1 \mathrm{M}$ sodium-citrate buffer (pH 4.5)+ HFD treatment,} Intraperitoneal injection for STZ and HFD orally

II Experimental control (EC)

III Placebo of SNEDDS formulation (P-SNEDDS) Placebo SNEDDS (without drugs), p.o

IV Placebo of nanosuspension formulation (P-NS) Placebo nanosuspension (without drugs), p.o

V Unprocessed GLM (U-GLM)

2mg (GLM), p.o

VI $\quad$ Unprocessed SIM (U-SIM)

$5 \mathrm{mg}(\mathrm{SIM})$, p.o

VII Unprocessed GLM-SIM (U-GLM-SIM)

$2 \mathrm{mg}(\mathrm{GLM})+5 \mathrm{mg}(\mathrm{SIM})$, p.o

VIII Nanosuspension GLM formulation at high dose 2mg (GLM), p.o (NS-GLM-H)

IX Nanosuspension GLM formulation at low dose $1 \mathrm{mg}(\mathrm{GLM})$, p.o (NS-GLM-L)

X Nanosuspension SIM formulation at high dose $5 \mathrm{mg}(\mathrm{SIM})$, p.o (NS-SIM-H)

XI Nanosuspension SIM formulation at low dose $2.5 \mathrm{mg}(\mathrm{SIM})$, p.o (NS-SIM-L)

XII Nanosuspension GLM-SIM formulation (NS- $\quad 1 \mathrm{mg}(\mathrm{GLM})+2.5 \mathrm{mg}(\mathrm{SIM})$, p.o GLM-SIM)

XIII SNEDDS GLM formulation at high dose 2mg (GLM), p.o (SNEDDS-GLM-H)

XIV SNEDDS GLM formulation at low dose $\quad 1 \mathrm{mg}(\mathrm{GLM})$, p.o (SNEDDS-GLM-L)

XV SNEDDS SIM formulation at high dose $\quad 5 \mathrm{mg}(\mathrm{SIM})$, p.o (SNEDDS-SIM-H)

XVI SNEDDS SIM formulation at low dose (SNEDDS- $2.5 \mathrm{mg}(\mathrm{SIM})$, p.o SIM-L)

XVII SNEDDS GLM-SIM formulation (SNEDDS-GLM- $\quad 1 \mathrm{mg}(\mathrm{GLM})+2.5 \mathrm{mg}(\mathrm{SIM})$, p.o SIM) 
After the administration of HFD for 14 days and STZ, all the animals (except group I) received their respective treatment from day 18th as indicated in Table 1 and continued daily for a period of 4 weeks (i.e., upto 46th day). The biochemical parameters, oxidative biomarkers, and histopathology were performed $24 \mathrm{~h}$ after administration of last dose at 46th day. The study protocol is shown in Fig. 1.

\subsubsection{Biochemical studies}

For determination of biochemical parameters blood was withdrawn for retro-orbital plexus and collected in plain Eppendorf tubes. Blood samples were processed to separate serum and it was stored in the deep freezer before biochemical analysis. Kits of Erba Diagnostics, India was used to estimate the biochemical parameters (Garg et al. 2019, Garg et al. 2017). Blood glucose level was determined using GOD-POD method. Serum levels of alkaline phosphatase (ALP), aspartate aminotransferase (AST), alanine transaminase (ALT) was estimated to check the liver function of rats of each group. Serum lipid profiles were determined by measuring triglycerides (TG), total cholesterol (TC) and high-density lipoprotein (HDL).

\subsubsection{Determination of in vivo antioxidants}

The kidneys have been separated, weighed and washed for blood removal in ice-cold saline. Separated kidneys were cut into parts and homogenized (Glass-Teflon pot-ter homogenizer, Thomas Scientific, USA) using pH 7.5 buffer $(0.025 \mathrm{M}$ Tris- $\mathrm{HCl})$. Homogenate tissue was centrifuged for 10 min at $4^{\circ} \mathrm{C}$ at 10,000 $\mathrm{rpm}$. The supernatant was isolated and used for different estimates of antioxidant enzymes. Liver homogenates were prepared in ice-cold $10 \%(\mathrm{w} / \mathrm{v})$ potassium chloride solution, levels and activities of various markers were measured. These include - catalase (CAT) (Sinha 1972), lipid peroxidation (LPO) (Ohkawa et al. 1979), and reduced glutathione (GSH) (Ellman 1959).

\subsubsection{Histopathological studies}

Paraffin sections of kidney and liver tissues were made and stained with haematoxylin and eosin to observe histopathological changes on 400X under microscope (Garg et al. 2017).

\subsubsection{Statistical analysis}

All the experimental data are expressed as mean \pm standard deviation (SD). Statistical assessment of the acquired information was performed using analysis of variance or Tukey's multiple comparison test using GraphPad Prism version 7.0 (GraphPad Software Inc., CA, USA). The P $<0.05$ value showed a substantial difference in the outcomes achieved.

\section{Results And Discussion}

The formulation development, its characterization and pharmacokinetic studies have been reported in the recent publication (Pandey et al. 2020). It was observed that the particle size of SP-NS was found to be $127.4 \pm 1.13 \mathrm{~nm}$ with PDI $0.265 \pm 0.05$. The optimized L-SNEDDS average droplet size was $55.63 \pm 1.78 \mathrm{~nm}$ with PDI of $0.235 \pm 0.06$. Similarly, average droplet size of S-SNEDDS was $75.26 \pm 2.38 \mathrm{~nm}$ with PDI of 
$0.271 \pm 0.09$. The zeta potential for SP-NS, L-SNEDDS and S-SNEDDS were found to be $-27.32 \pm 2.05 \mathrm{mV}$, $-22.31 \pm 1.66 \mathrm{mV}$, and $-19.54 \pm 1.56 \mathrm{mV}$, respectively. The drug loading (\%) of GLM in SP-NS, L-SNEDDS and S-SNEDDS were found to be $95.25 \pm 4.32,94.5 \pm 2.74$, and $95.5 \pm 3.23$, respectively. Similarly, the drug loading (\%) of SIM in SP-NS, L-SNEDDS and S-SNEDDS were found to be $80.30 \pm 2.44,79.2 \pm 1.94$, and $92.63 \pm 4.45$, respectively. The dissolution study indicated significantly $(p<0.05)$ faster release of GLM and SIM from L-SNEDDS, S-SNEDDS and SP-NS than their unprocessed forms. The permeability studies conducted using Caco-2 cell monolayer indicated about 2.37, 1.84- and 2.69-folds increase in drug permeation for GLM and 2.52, 2.08- and 2.78-folds increase for SIM from L-SNEDDS, S-SNEDDS and SPNS respectively as compared to their unprocessed form. Similarly, the pharmacokinetic studies carried on rats revealed that the total plasma concentration of both the drugs in SNEDDS and SP-NS were significantly $(P<0.05)$ higher than that of their unprocessed form. About 6.69 - and 4.22 -folds increase in the area under the curves (AUCs) of GLM present in nanosuspensions and SNEDDS and about 1.76- and 2.68-folds increase AUCs of SIM present in nanosuspensions and SNEDDS were reported as compared to their unprocessed forms. Interestingly, the results indicated that the AUCs of nanosuspensions of GLM and SIM were 1.59 and 1.52 higher than SNEDDS respectively (Pandey et al. 2020). These positive outcomes of aforementioned studies helped to initiate the pharmacodynamic studies for the formulations on high fat diet and streptozotocin induced rat model.

\subsection{Effects on body weight}

Although there are different animal models available for the study of type 2 diabetes (spontaneous as well as induced), the pattern of disease initiation and progression in most of them does not seem to be closely comparable to the human clinical situation. High-fat diet and streptozotocin-induced model would, on the one hand, closely resemble the natural history of disease events (from insulin tolerance to beta-cell dysfunction) as well as the metabolic features of human type 2 diabetes and, on the other hand, be simpler, easier to use and useful for analysis and preclinical testing of different compounds for the treatment of type 2 diabetes. The materialization of the pattern of disease was achieved by combining HFD feeding which developed insulin resistance and low dose of STZ treatment which caused the initial beta-cell dysfunction and subsequently the frank hyperglycaemia in non-genetic, outbred Sprague Dawley rats (Srinivasan et al. 2005).

The changes in average body weights of each group after giving HFD for 15 days and change in body weight after treatment are shown in Fig. 2. Results clearly indicated that the body weight of each group was significantly increasing with HFD and decline in body weight was observed after induction of STZ till the start of treatment (16th-17th day). Gradual recovery was observed in rats receiving U-GLM, U-SIM, UGLM-SIM, NS-GLM-H, NS-GLM-L, NS-SIM-H, NS-SIM-L, SNEDDS-GLM-H, SNEDDS-GLM-L, SNEDDS-SIM-H and SNEDDS-SIM-L as compared to normal control (GI). No recovery was observed in rats receiving EC, PSNEDDS, and P-NS. These results also suggested the effectiveness of the formulation for improvement of loss in body weight in diabetic animals receiving treatment of drugs and nanoformulations, however, immediate and significant increase in body weight was found in rats receiving NS-GLM-SIM and SNEDDS-GLM-SIM during the treatment, who received nanosuspensions and SNEDDS of GLM-SIM 
mixture respectively as compared to other formulations. The significant elevation of body weight in type 2 diabetic rats when compared to NC rats could be attributed to the dietary regimen of HFD and STZ administration. However, the hyperglycaemic state in type 2 diabetic control rats were gradually suppressed in groups treated with formulations having GLM-SIM mixture. The observed decrease in blood glucose levels after treatment with formulation may suggest the antihyperglycemic properties in vivo.

\subsection{Effect on blood glucose level}

The changes in blood glucose level of different groups after receiving HFD, STZ and treatment through different formulation of GLM, SIM and GLM-SIM mixture are shown in Fig. 3. There is no change in blood glucose level (160-180 mg/dL) was found with HFD, however, sudden increase in glucose level varying from $370-390 \mathrm{mg} / \mathrm{dL}$ were estimated administration of STZ (i.v. $35-50 \mathrm{mg} / \mathrm{kg}$ ) to each group. Significant decrease in blood glucose level was observed in rats receiving NS-GLM-SIM, SNEDDS-GLM-SIM, NS-GLM$\mathrm{H}$, SNEDDS-GLM-H, NS-GLM-H, SNEDDS-GLM-H, NS-GLM-L, SNEDDS-GLM-L, U-GLM-SIM, and U-GLM as compared to EC. Little difference in glucose level was found in rats receiving NS-GLM-SIM as compared to $\mathrm{NC}$, which confirmed the significant improvement in diabetic condition after receiving nanosuspensions of GLM-SIM mixture. This result also established the effectiveness of the formulations in given order- NS-GLM-SIM > SNEDDS-GLM-SIM > NS-GLM-H > SNEDDS-GLM-H > NS-GLM-H > SNEDDSGLM-H > NS-GLM-L > SNEDDS-GLM-L > U-GLM-SIM > U-GLM

\subsection{Effect on biochemical parameters}

Table 2 shows the change in biochemical parameters such as ALP, creatinine and urea, in each group after the treatment. It is observed that there was no significant change in all the biochemical parameters (ALP, creatinine and urea) in rats receiving NS-GLM-SIM as compared to NC. Significant differences in level of these biochemical parameters were observed in groups P-SNEDDS to SNEDDS-GLM-SIM as compared to EC rats. However, maximum improvements in level of biochemical parameters were observed in rats receiving NS-GLM-SIM. These results directly indicated the effectiveness of formulation received by NS-GLM-SIM. 
Table 2

Biochemical Parameters of each group

\begin{tabular}{|c|c|c|c|}
\hline Groups & $\operatorname{ALP}(\mathrm{U} / \mathrm{dL})$ & Creatinine $(\mathrm{mg} / \mathrm{dL})$ & Urea (mg/dL) \\
\hline NC & $32.18 \pm 3.16$ & $0.25 \pm 0.09$ & $22.45 \pm 3.32$ \\
\hline EC & $92.56 \pm 2.56^{* \star \star a}$ & $0.94 \pm 0.05,^{* \star \star a}$ & $58.56 \pm 4.48^{* \star \star a}$ \\
\hline P-SNEDDS & $83.44 \pm 3.78^{* \star \star a,{ }^{\star} \beta}$ & $0.90 \pm 0.68,{ }^{* \star \star a}$ & $51.66 \pm 2.45^{* \star \star} a,{ }^{* \star \beta}$ \\
\hline P-NS & $78.16 \pm 2.00^{* \star \star a} a^{* \star \star \beta}$ & $0.86 \pm 0.08,{ }^{* \star \alpha} a$ & $57.11 \pm 3.35^{\star \star \star a}$ \\
\hline U-GLM & $67.12 \pm 2.98^{\star \star \star} a,{ }^{* \star \star} \beta$ & $0.75 \pm 0.04,^{* \star \star a}$ & $38.55 \pm 2.22^{\star \star \star a,{ }^{* \star \star} \beta}$ \\
\hline U-SIM & $72.21 \pm 3.00^{* \star \star a} a, \star \star \beta \beta$ & $0.81 \pm 0.09,{ }^{* \star \star a}$ & $42.03 \pm 3.31^{* \star \star} a,{ }^{* \star \star} \beta$ \\
\hline U-GLM-SIM & $62.78 \pm 4.22^{\star \star \star} a,{ }^{* \star \star} \beta$ & $0.77 \pm 0.04,{ }^{* \star \star a}$ & $37.21 \pm 1.26^{\star \star \star} a,{ }^{* \star \star} \beta$ \\
\hline NS-GLM-H & $51.04 \pm 2.25^{* \star \star a},{ }^{* \star \star \beta} \beta$ & $0.46 \pm 0.07,{ }^{* \star \beta}$ & $31.12 \pm 3.12^{\star \star \star} a,{ }^{* \star \star} \beta$ \\
\hline NS-GLM-L & $46.62 \pm 4.56^{\star \star \star a},{ }^{\star \star \star \star} \beta$ & $0.39 \pm 0.02,{ }^{* \star \star \beta}$ & $29.43 \pm 2.22^{* \star a},{ }^{\star \star \star} \beta$ \\
\hline NS-SIM-H & $67.34 \pm 1.09^{\star \star \star} a,{ }^{* \star \star} \beta$ & $0.55 \pm 0.08,{ }^{\star} \beta$ & $38.39 \pm 1.98^{* \star \star} a,{ }^{* \star \star \beta}$ \\
\hline NS-SIM-L & 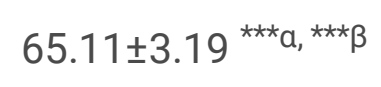 & $0.51 \pm 0.05^{, * \star \beta}$ & 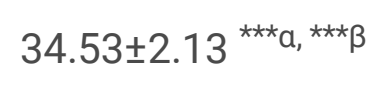 \\
\hline NS-GLM-SIM & $40.01 \pm 3.49^{\star \star \star \beta}$ & $0.29 \pm 0.07,{ }^{\star \star \star \beta}$ & $25.22 \pm 0.67^{\star \star \star \beta}$ \\
\hline SNEDDS-GLM-H & $58.20 \pm 5.54^{\star \star \star} \mathrm{a},{ }^{* \star \star} \beta$ & $0.50 \pm 0.02,{ }^{* \star \beta}$ & 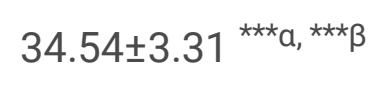 \\
\hline SNEDDS-GLM-L & $52.53 \pm 3.76^{\star \star \star a},{ }^{* \star \star \beta} \beta$ & $0.45 \pm 0.01,{ }^{* \star \beta}$ & $33.30 \pm 2.11^{\star \star \star} a,{ }^{* \star \star} \beta$ \\
\hline SNEDDS-SIM-H & $70.56 \pm 6.98^{\star \star \star a} a^{\star \star \star} \beta$ & $0.60 \pm 0.06$ & $34.65 \pm 3.22^{\star \star \star a},{ }^{* \star \star} \beta$ \\
\hline SNEDDS-SIM-L & $61.78 \pm 3.46^{\star \star \star} a_{,}{ }^{* \star \star} \beta$ & $0.54 \pm 0.08,{ }^{\star \beta}$ & $32.22 \pm 1.18^{\star \star \star a} a,{ }^{\star \star \star} \beta$ \\
\hline SNEDDS-GLM-SIM & $44.56 \pm 5.12^{\star \star \star \alpha},{ }^{\star \star \star} \beta$ & $0.33 \pm 0.05^{, \star \star \star \beta}$ & $28.19 \pm 3.26^{* a}{ }^{\star \star \star \star} \beta$ \\
\hline
\end{tabular}

\subsection{Effect on antioxidant levels}

The effect of treatment on GSH level of each group is shown in Fig. 4a. Fast recovery in GSH level was found in rats receiving NS-GLM-SIM and SNEDDS-GLM-SIM after the treatment. No significant difference in GSH level is observed in rats receiving NS-GLM-SIM and SNEDDS-GLM-SIM as compared to NC rats and significant difference was observed in both groups as compared to EC rats. Effect of treatment on level of CAT and TBARS level of each group are shown in Figs. 4b and 4c, respectively. The rats receiving NS-GLM-SIM and SNEDDS-GLM-SIM showed better result as compared to other groups because level of 
these three parameters were found same as in group I (NC) and significant different as compared to group II (EC). The results of rats receiving NS-GLM-SIM were found much improved as that of rats receiving SNEDDS-GLM-SIM. Type 2 diabetes mellitus (T2DM) is a heterogeneous metabolic syndrome that is due to a range of pathophysiological mechanisms while insulin resistance (IR) and $\beta$-cell failure play key roles. In a wide range of degenerative diseases including cancer, cardiovascular disorders, diabetes and its complications, free radicals and reactive oxygen species (ROS) have been involved. Growing evidence suggested that oxidative stress increases during diabetes due to overproduction of ROS and decreased antioxidant defense capacity, which starts very early and worsens throughout disease. There are several studies which show that there could be weaker antioxidant defenses in diabetes. These include records of decreased plasma / serum overall antioxidant status or free radical scavenging activity, and increased plasma oxidation in type 2 diabetes, along with reduced levels of common antioxidants such as ascorbic acid and vitamin E. Furthermore, the activities of the antioxidant enzymes catalase, superoxide dismutase and glutathione peroxidase were identified as reduced in diabetes (Bajaj \&Khan 2012, Hadi et al. 2015, Rahimi-Madiseh et al. 2016, Verma et al. 2013). Our results showed a significant improvement in the levels of serum lipids and oxidative stress markers in patients with T2DM and dyslipidaemia treated with glimepiride and simvastatin loaded nanoformulations. There are several evidence suggests there is a close positive association between hyperlipidemia and increased oxidative damage.

\subsection{Effect on SGOT and SGPT level}

Figure 5a. shows the effect of treatment on SGOT level of each group. There was no significant in level of SGOT found in rats receiving NS-GLM-H, NS-GLM-SIM, SNEDDS-GLM-H and SNEDDS-GLM-SIM as compared to NC rats. However, significant differences in SGOT level were observed in same group. These studies indicated the effectiveness of the formulation nanosuspensions and SNEDDS of GLM-SIM mixture received by rats of groups NS-GLM-SIM and SNEDDS-GLM-SIM, respectively. Different observations in level of SGPT were found and it was shown in Fig. 5b. Level of SGPT was estimated same in rats of groups NS-GLM-SIM as in NC and significant difference in their SGPT level was observed as compared to rats of $\mathrm{NC}$ group. These observations clearly indicated the superiority of the formulation administered to rats receiving nanosuspensions of GLM-SIM mixture (NS-GLM-SIM).

\subsection{Effect on lipid level}

Lipid level of each group was estimated at 1st, 18th, and 46th day and results are shown in Fig. 6a-d. Sudden increment in TG, LDL and total cholesterol level in all groups were observed at 18th day due the high fatty diet. Immediate and significant lowering in level of these lipids was recorded in group XII and XVII after the treatment (46th day). A different trend was observed in HDL. Its level was drastically decreased in each group after high fatty diet. However, HDL level in group XII and XVII were significantly raised and found similar with group I (Normal control).

\subsection{Histopathological studies}


The images of kidney and liver sections of rats of different groups are shown in Fig. 7 and 8, respectively. In case of NC rats normal tissue architecture was observed. The rats of EC, P-SNEDDS, P-NS complete cellular damage. The cellular architecture was found to be improved in case of rats receiving U-GLM, USIM, U-GLM-SIM, NS-GLM-L, NS-GLM-H, NS-SIM-L, NS-SIM-H, NS-GLM-SIM, SNEDDS-GLM-H, SNEDDSGLM-L, SNEDDS-SIM-L, SNEDDS-SIM-H and SNEDDS-GLM-SIM. The recovery was found in the following increasing order:

U-SIM $<$ U-GLM $<$ U-GLM-SIM $<$ SNEDDS-SIM-L < NS-SIM-L<SNEDDS-SIM-H $<$ NS-SIM-H $<$ SNEDDS-GLM-L $<$ NS-GLM-L< SNEDDS-GLM-H< NS-GLM-H< SNEDDS-GLM-SIM $<$ NS-GLM-SIM

General observations are given below:

Figure 7: Kidney - NC: Architecture of the tissue was found normal; EC, P-SNEDDS and P-NS: Severe tubular epithelial atrophy, mesangial proliferation, sclerotic changes in the glomerulus. Congestion of capillaries very close to the normal tissue structure.; U-GLM: Reduction in tubular epithelial atrophy, mesangial proliferation and sclerotic changes in the glomerulus but lesser than U-GLM-SIM; U-SIM: Reduction in tubular epithelial atrophy, mesangial proliferation and sclerotic changes in the glomerulus but lesser than U-GLM; U-GLM-SIM: Reduction in tubular epithelial atrophy, mesangial proliferation and sclerotic changes in the glomerulus but lesser than SNEDDS-SIM-L; NS-GLM-H: Protection of tubular epithelial cells and congestion of capillaries was observed which was lesser; NS-GLM-L: Protection of tubular epithelial cells and congestion of capillaries was observed which was lesser than NS-GLM-H; NSSIM-H: Lesser atrophy in epithelial cells and congestion of capillaries was observed; NS-SIM-L: Lesser atrophy in epithelial cells and congestion of capillaries was observed but less than NS-SIM-H; NS-GLMSIM: Showed highest regeneration in tubular epithelial cells and very less congestion of capillaries; SNEDDS-GLM-H: Protection of tubular epithelial cells and congestion of capillaries was observed which was lesser than NS-GLM-SIM, SNEDDS-GLM-SIM, NS-GLM-H and NS-GLM-L; SNEDDS-GLM-L: Protection of tubular epithelial cells and congestion of capillaries was observed but lesser than SNEDDS-GLM-H; SNEDDS-SIM-H: Lesser atrophy in epithelial cells and congestion of capillaries was observed but less than NS-SIM-H; SNEDDS-SIM-L: Reduction in tubular epithelial atrophy, mesangial proliferation and sclerotic changes in the glomerulus but less than SNEDDS-SIM-H; SNEDDS-GLM-SIM: More protection was observed in tubular epithelial cells and capillaries but less than NS-GLM-SIM.

Figure 8: Liver: NC: Normal hepatic cells with well-preserved cytoplasm, nucleus, nucleolus, and central vein; EC, P-SNEDDS and P-NS: Liver section showed that the lobular architecture was maintained, but there was also severe fatty change, sinusoidal dilation, severe feathery degeneration and necrosis. UGLM: Showed less regeneration of hepatocytes and fatty changes less than U-GLM-SIM; U-SIM: Showed less regeneration of hepatocytes and fatty changes less than U-GLM; U-GLM-SIM: Showed less regeneration of hepatocytes and fatty changes SNEDDS-SIM-L; NS-GLM-H: Showed high regeneration of hepatocytes with less fatty changes. Sinusoidal dilation was observed lesser than SNEDDS-GLM-SIM; NS-GLM-L: Showed high regeneration of hepatocytes with less fatty changes. Sinusoidal dilation was observed lesser than NS-GLM-H; NS-SIM-H: Showed regeneration of hepatocytes with less fatty changes 
but less effective with respect to SNEDDS-GLM-L; NS-SIM-L: Showed regeneration of hepatocytes with less fatty changes but less effective with respect to NS-SIM-H; NS-GLM-SIM: Showed highest regeneration of hepatocytes; SNEDDS-GLM-H: Showed regeneration of hepatocytes with less fatty changes but the effect was lesser than NS-GLM-SIM, SNEDDS-GLM-SIM, NS-GLM-H and NS-GLM-L; SNEDDS-GLM-L: Showed regeneration of hepatocytes with Less fatty changes but effect was lesser than SNEDDS-GLM-H; SNEDDS-SIM-H: Showed regeneration of hepatocytes and less fatty changes were observed but less than NS-SIM-H; SNEDDS-SIM-L: Showed regeneration of hepatocytes with less sinusoidal dilation but the effect was less than SNEDDS-SIM-H; SNEDDS-GLM-SIM: Showed high regeneration of hepatocytes. Less fatty changes and sinusoidal dilation was observed but less than NSGLM-SIM.

The results clearly indicated that the nanosuspension containing both the drugs have shown better recovery of cells as compared to their SNEDDS formulations. This could be due to better permeability, Cmax and AUC of drugs loaded in nanosuspension with respect to SNEDDS as reported in previous investigation (Pandey et al. 2020).

\section{Conclusion}

The study entailed successful development of two different nanoarchitectures viz. S-SNEDDS and SP-NS. Both the formulations have shown optimised physicochemical characteristics and improvement in the biochemical parameters including the histopathology of liver and kidney of rats undergone treatment as compared to their unprocessed form (U-GLM and U-SIM). This indicated that the drugs were found to be more effective when they are delivered through nanocarriers. It is important to note that the combination of both the drugs was found to be more effective than their individual doses. The in-vivo results indicated that nanosuspension containing both the drugs (NS-GLM-SIM) was found more efficacious than that of their SNEDDS formulation (SNEDDS-GLM-SIM). The positive results of pre-clinical studies indicated towards exploration of these formulations for clinical trials so that the research should reach in the form of product behind bedside of patients suffering from T2DM.

\section{Declarations}

\section{Authors' Contributions:}




\begin{tabular}{|lll|}
\hline S.N. & Authors & CRediT role(s) \\
\hline $\mathbf{1}$ & $\begin{array}{l}\text { Narendra Kumar } \\
\text { Pandey }\end{array}$ & Methodology, Data Curation and Writing - original draft \\
\hline $\mathbf{3}$ & Bimlesh Kumar & $\begin{array}{l}\text { Methodology } \\
\text { editing }\end{array}$ \\
\hline $\mathbf{4}$ & Monica Gulati & Review \& editing \\
\hline $\mathbf{5}$ & Sukriti Vishwas & Review \& editing \\
\hline $\mathbf{6}$ & Rubiya Khursheed & Review \& editing \\
\hline $\mathbf{7}$ & Harish Dureja & Methodology \\
\hline $\mathbf{8}$ & $\begin{array}{l}\text { Dinesh Kumar } \\
\text { Chellappan }\end{array}$ & Methodology \\
\hline $\mathbf{9}$ & Niraj Kumar Jha & Methodology \\
\hline 10 & Ankur Sharma & Methodology \\
\hline 11 & Saurabh Kumar Jha & Methodology \\
\hline 12 & Piyush Kumar Gupta & Methodology \\
\hline 13 & Saurabh Gupta & Methodology \\
\hline 14 & Gaurav Gupta & Methodology \\
\hline 15 & Parteek Prasher & Methodology \\
\hline 16 & Kamal Dua & Review \& editing \\
\hline
\end{tabular}

Acknowledgement: Authors are thankful to Central Instrumentation Facility, Lovely Professional University for providing necessary support in terms of sample analysis using sophisticated instruments such as DSC, PXRD, SEM and HPLC.

Ethics Approval and Consent to Participate: Not Applicable

Consent for Publication: Yes

Availability of Data and Materials: Not Applicable

Competing Interests: None

Funding: This research did not receive any specific grant from funding agencies in the public, commercial, or not-for-profit sectors. 


\section{References}

1. Bajaj S, Khan A (2012) Antioxidants and diabetes. Indian J Endocrinol Metabol 16:S267

2. Basit A, Riaz M, Fawwad A (2012) Glimepiride: evidence-based facts, trends, and observations. Vasc Health Risk Manag 8:463

3. Cho N, Shaw J, Karuranga S, Huang Y, da Rocha Fernandes J, Ohlrogge A, Malanda B (2018) IDF Diabetes Atlas: Global estimates of diabetes prevalence for 2017 and projections for 2045. Diabetes Res Clin Pract 138:271-281

4. Ellman GL (1959) Tissue sulfhydryl groups. Arch Biochem Biophys 82:70-77

5. Garg V, Kaur P, Singh SK, Kumar B, Bawa P, Gulati M, Yadav AK (2017) Solid self-nanoemulsifying drug delivery systems for oral delivery of polypeptide-k: Formulation, optimization, in-vitro and in-vivo antidiabetic evaluation. Eur J Pharm Sci 109:297-315

6. Garg V, Kaur P, Gulati M, Singh SK, Kumar B, Pandey NK, Yadav AK, Kumar R, Kuppusamy G, De A (2019) : Coadministration of Polypeptide-k and Curcumin Through Solid Self-Nanoemulsifying Drug Delivery System for Better Therapeutic Effect Against Diabetes Mellitus: Formulation, Optimization, Biopharmaceutical Characterization, and Pharmacodynamic Assessment. Assay and drug development technologies

7. Gazzerro P, Proto MC, Gangemi G, Malfitano AM, Ciaglia E, Pisanti S, Santoro A, Laezza C, Bifulco M (2012) Pharmacological actions of statins: a critical appraisal in the management of cancer. Pharmacol Rev 64:102-146

8. Ghosh D, Singh SK, Khursheed R, Pandey NK, Kumar B, Kumar R, Kumari Y, Kaur G, Clarisse A, Awasthi A (2020) Impact of solidification on micromeritic properties and dissolution rate of selfnanoemulsifying delivery system loaded with docosahexaenoic acid. Drug Development and Industrial Pharmacy, pp 1-33

9. Hadi NR, Abdelhussein MA, Rudha AR, Jamil DA, Al-Aubaidy HA (2015) Simvastatin use in patients with type 2 diabetes mellitus: the effects on oxidative stress. Oman medical journal 30:237

10. Inugala S, Eedara BB, Sunkavalli S, Dhurke R, Kandadi P, Jukanti R, Bandari S (2015) Solid selfnanoemulsifying drug delivery system (S-SNEDDS) of darunavir for improved dissolution and oral bioavailability: in vitro and in vivo evaluation. Eur J Pharm Sci 74:1-10

11. Karuranga S, da Rocha Fernandes HY, Malanda B (2018) : IDF Diabetes Atlas 8th Edition. International Diabetes Federation

12. Khursheed R, Singh SK, Wadhwa S, Kapoor B, Gulati M, Kumar R, Ramanunny AK, Awasthi A, Dua K (2019) : Treatment strategies against diabetes: Success so far and challenges ahead.European journal of pharmacology, 172625

13. Khursheed R, Singh SK, Wadhwa S, Gulati M, Awasthi A, Kumar R, Ramanunny AK, Kapoor B, Kumar $P$, Corrie $L$ (2020) Exploring role of probiotics and Ganoderma lucidium extract powder as solid carriers to solidify liquid self-nanoemulsifying delivery systems loaded with curcumin. Carbohydrate Polymers, p 116996 
14. Kumar B, Garg V, Singh S, Pandey NK, Bhatia A, Prakash T, Gulati M, Singh SK (2018) Impact of spray drying over conventional surface adsorption technique for improvement in micromeritic and biopharmaceutical characteristics of self-nanoemulsifying powder loaded with two lipophilic as well as gastrointestinal labile drugs. Powder Technol 326:425-442

15. Mahesh KV, Singh SK, Gulati M (2014) A comparative study of top-down and bottom-up approaches for the preparation of nanosuspensions of glipizide. Powder Technol 256:436-449

16. Ohkawa H, Ohishi N, Yagi K (1979) Assay for lipid peroxides in animal tissues by thiobarbituric acid reaction. Anal Biochem 95:351-358

17. Pandey NK, Singh SK, Gulati M, Kumar B, Kapoor B, Ghosh D, Kumar R, Khursheed R, Awasthi A, Kuppusamy G (2020) Overcoming the dissolution rate, gastrointestinal permeability and oral bioavailability of glimepiride and simvastatin co-delivered in the form of nanosuspension and solid self-nanoemulsifying drug delivery system: A comparative study, 102083 edn. Journal of Drug Delivery Science and Technology

18. Rahimi-Madiseh M, Malekpour-Tehrani A, Bahmani M, Rafieian-Kopaei M (2016) The research and development on the antioxidants in prevention of diabetic complications. Asian Pacific journal of tropical medicine 9:825-831

19. Rajesh SY, Singh SK, Pandey NK, Sharma P, Bawa P, Kumar B, Gulati M, Jain SK, Gowthamarajan K, Singh S (2018) Impact of various solid carriers and spray drying on pre/post compression properties of solid SNEDDS loaded with glimepiride: in vitro-ex vivo evaluation and cytotoxicity assessment. Drug Dev Ind Pharm 44:1056-1069

20. Sharma P, Singh SK, Pandey NK, Rajesh SY, Bawa P, Kumar B, Gulati M, Singh S, Verma S, Yadav AK (2018) Impact of solid carriers and spray drying on pre/post-compression properties, dissolution rate and bioavailability of solid self-nanoemulsifying drug delivery system loaded with simvastatin. Powder Technol 338:836-846

21. Singh SK, Srinivasan K, Gowthamarajan K, Singare DS, Prakash D, Gaikwad NB (2011) Investigation of preparation parameters of nanosuspension by top-down media milling to improve the dissolution of poorly water-soluble glyburide. Eur J Pharm Biopharm 78:441-446

22. Sinha AK (1972) Colorimetric assay of catalase. Anal Biochem 47:389-394

23. Srinivasan K, Viswanad B, Asrat L, Kaul C, Ramarao P (2005) Combination of high-fat diet-fed and low-dose streptozotocin-treated rat: a model for type 2 diabetes and pharmacological screening. Pharmacol Res 52:313-320

24. Usman B, Satija S, Sharma N, Mehta M, Vyas M, Khatik GL, Khurana N, Hansbro PM, Williams K, Dua K (2019) Recent Developments in Alpha-Glucosidase Inhibitors for Management of Type-2 Diabetes. An Update. Current pharmaceutical design

25. Verma S, Sagar N, Vats P, Shukla K, Abbas M, Banerjee M (2013) Antioxidant enzyme levels as markers for type 2 diabetes mellitus. Int J Bioassays 2:685-690

\section{Figures}




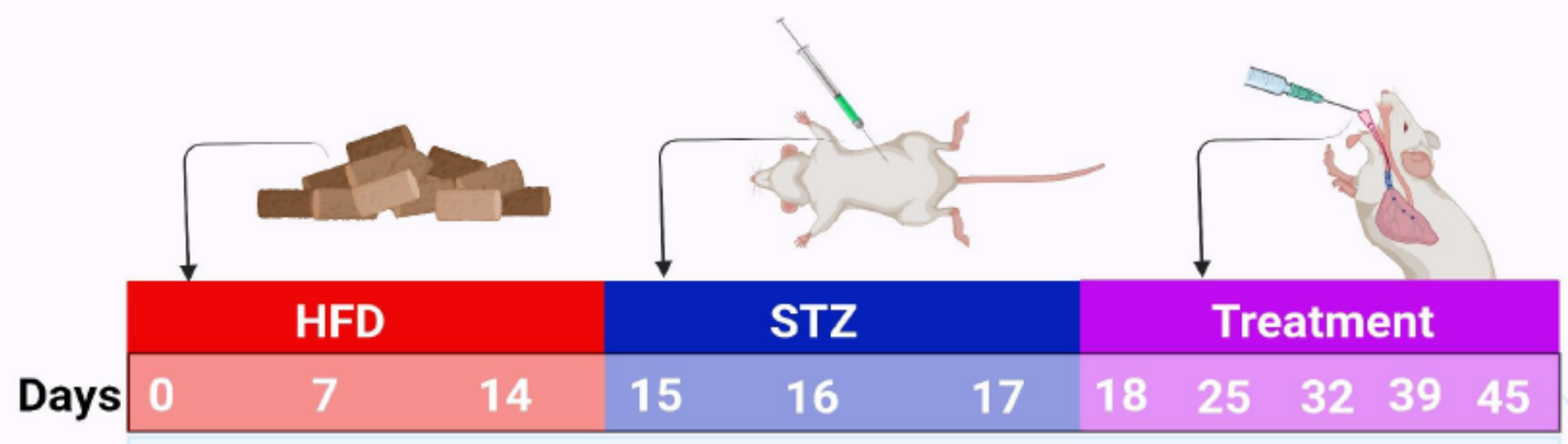

Measurement of body weight and Blood glucose level

Biochemical Parameters:

ALP, Creatinine, Urea, SGOT, SGPT, Total protein

Oxidative biomarkers:

GSH, Catalase and TBARS

Histopathology:

Liver and Kidney

Figure 1

Study procedure used for animal studies.

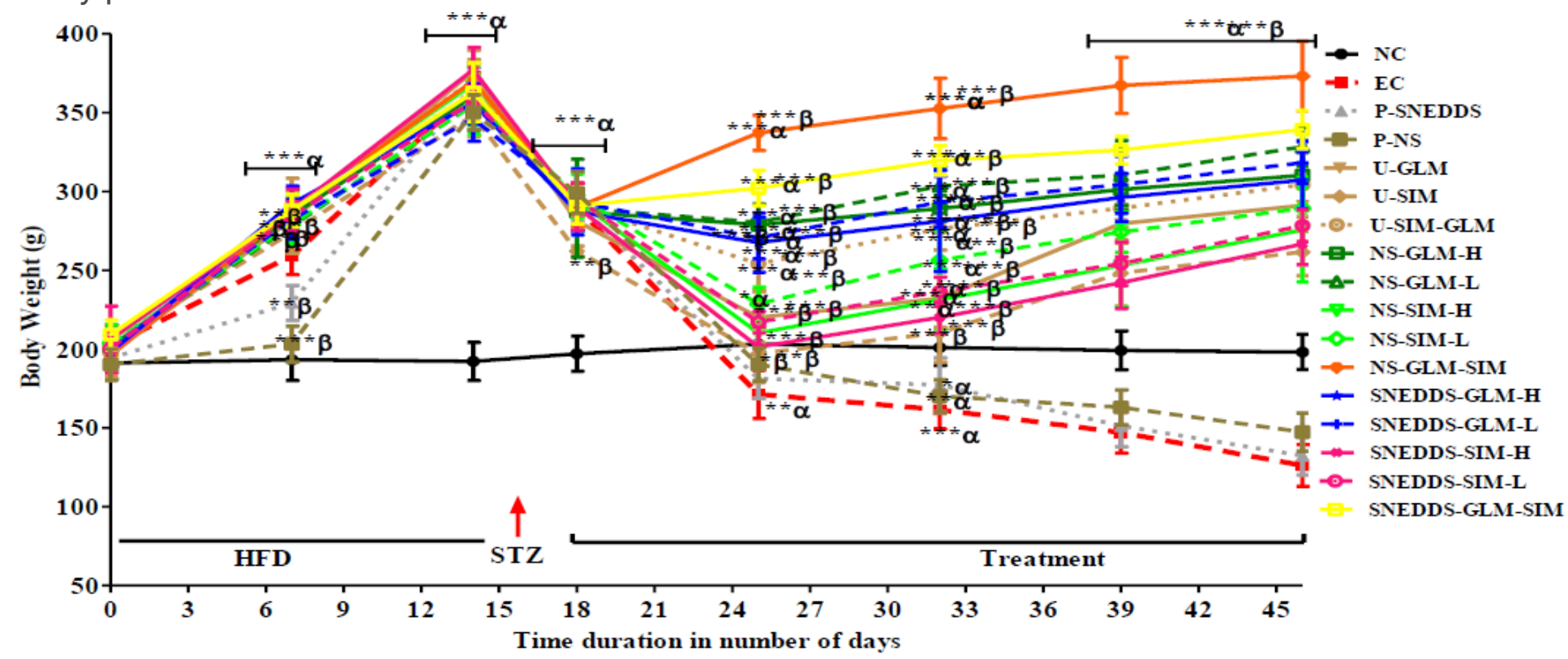

Figure 2 
Change in total body weight of each group. Note: a- comparison with $\mathrm{NC}, \beta$ - comparison with $\mathrm{EC}$, *- less difference $(p<0.05)$. ${ }^{*}$ - more difference $(p<0.001)$, ${ }^{* \star}$-significant difference $(p<0.0001)$

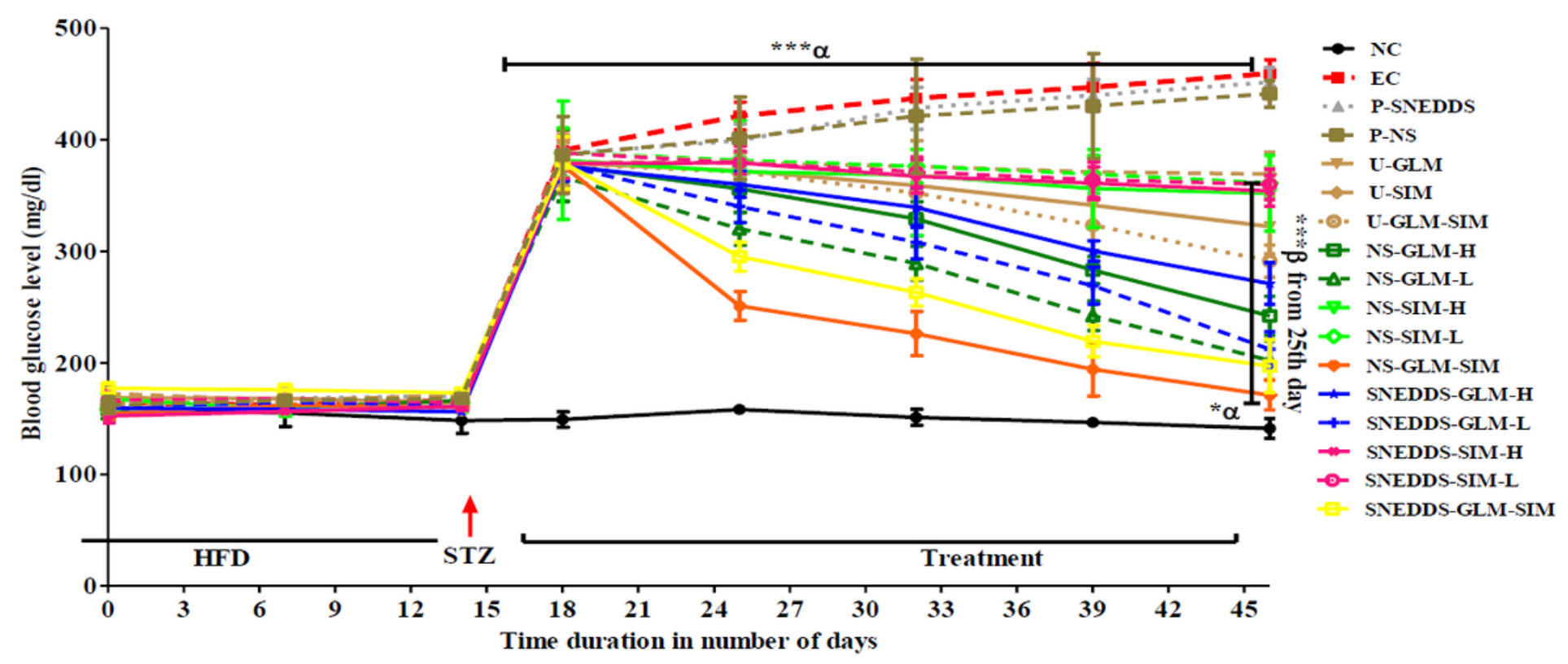

Figure 3

Effect of treatment on blood glucose level of each group. Note: $\alpha$ - comparison with $\mathrm{NC}, \beta$ - comparison with EC, ${ }^{*}$ - less difference $(p<0.05)$. **- more difference $(p<0.001)$, ${ }^{* *}$-significant difference $(p<0.0001)$. 

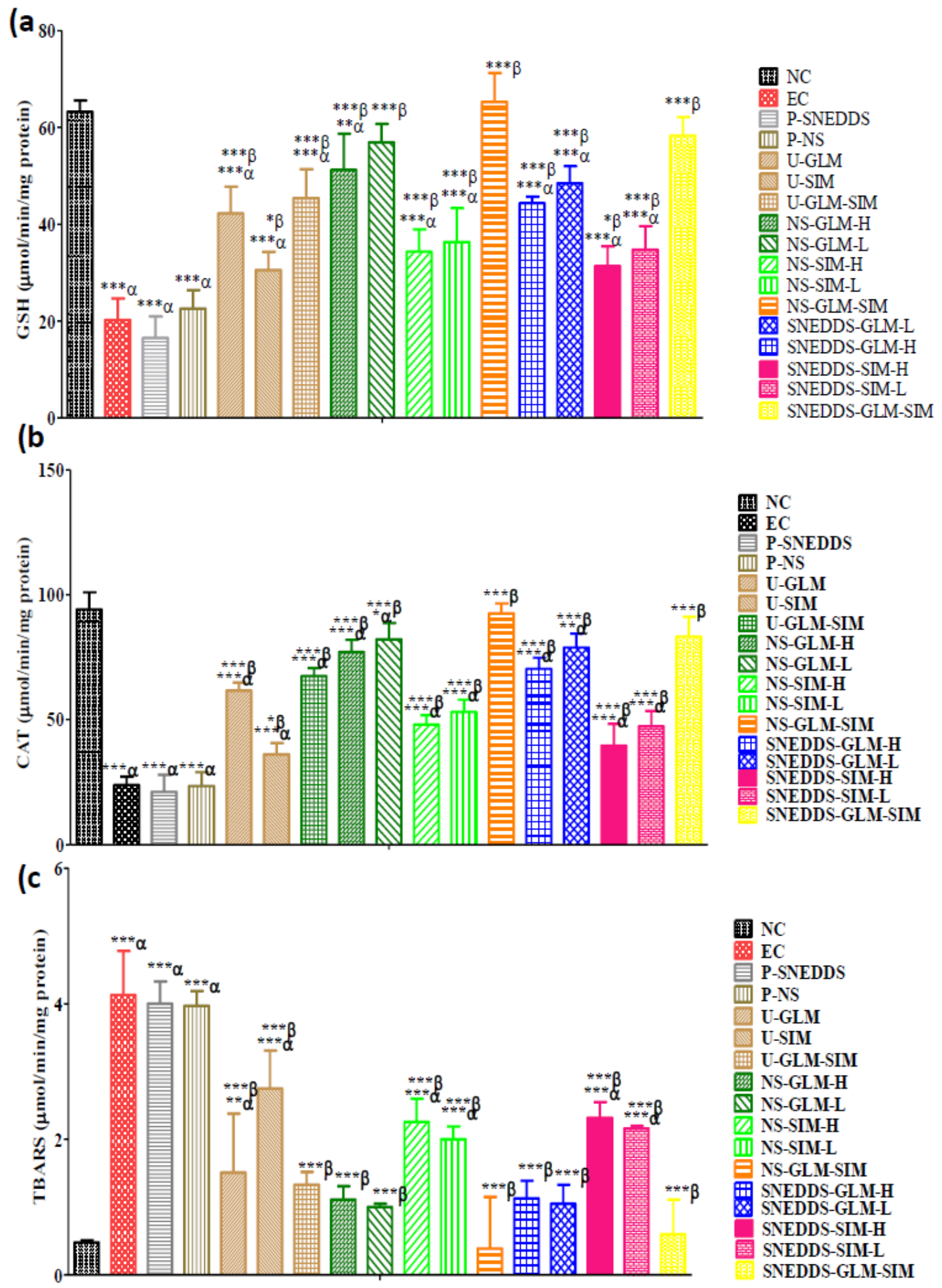

Figure 4

Effect of treatment on antioxidant levels of each group. a. GSH; b. CAT level; c. TBARS. Note: acomparison with NC, $\beta$ - comparison with $E C$, *- less difference $(p<0.05)$. **- more difference $(p<0.001)$, $* \star *_{-}$ significant difference $(p<0.0001)$. 

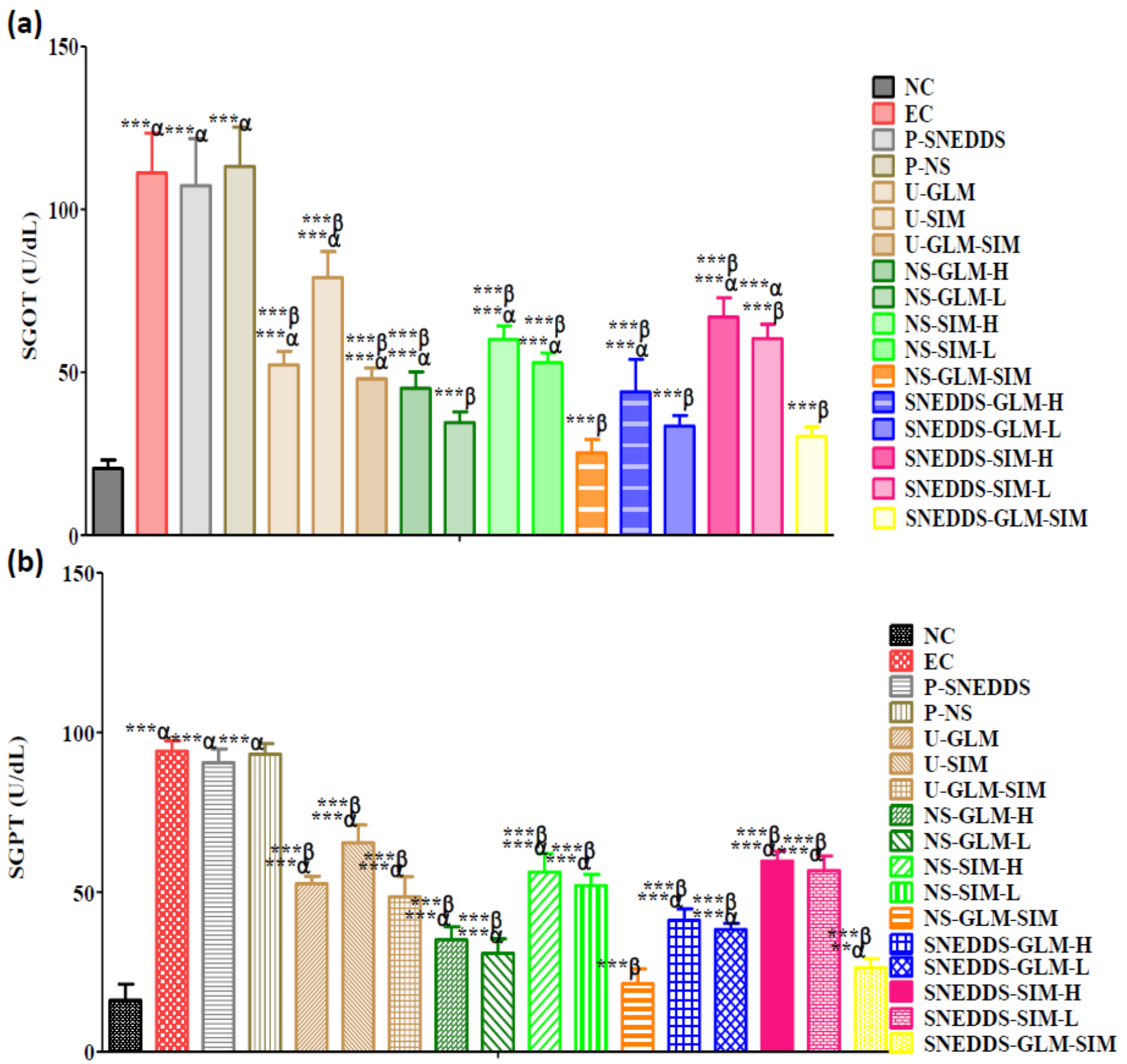

Figure 5

Effect of treatment on TG (a), HDL(b), LDL (c) and total cholesterol (d) levels of each group. Note: acomparison with NC, $\beta$ - comparison with $E C$, *- less difference $(p<0.05)$. **- more difference $(p<0.001)$, ***significant difference $(p<0.0001)$ 
(a)
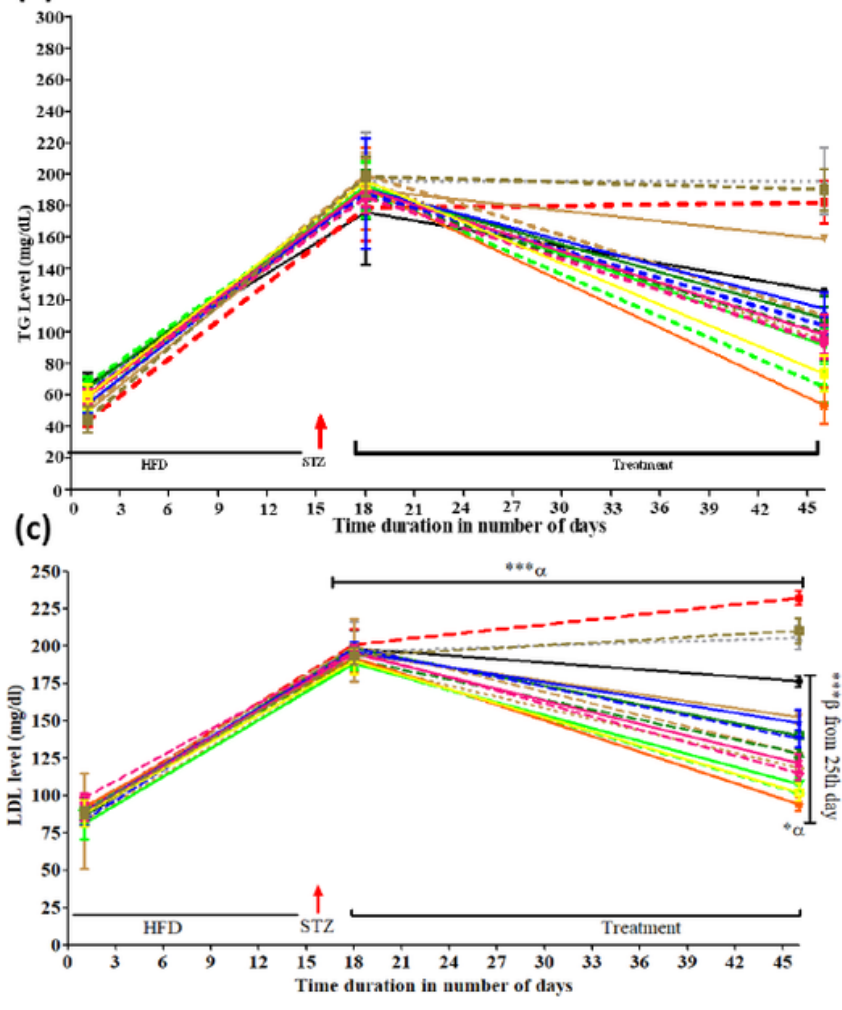

(b)

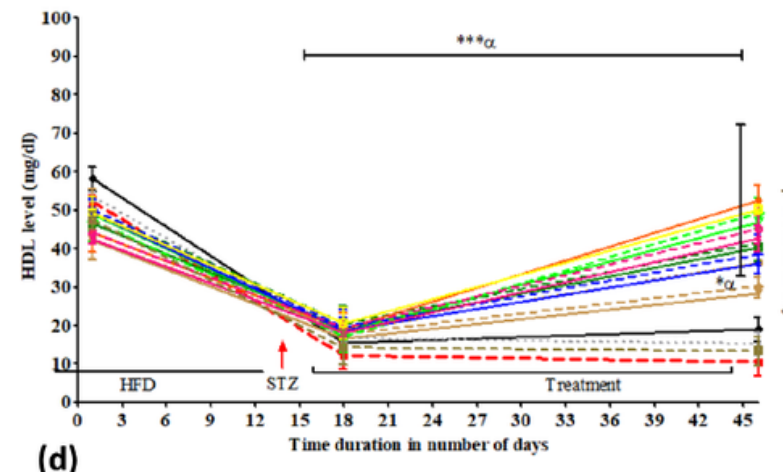

(d)

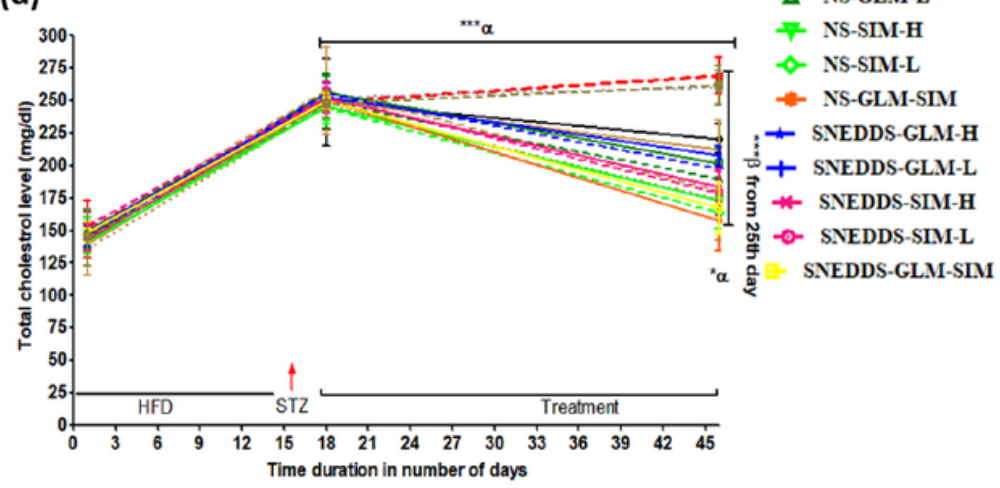

Figure 6

Effect of treatment on SGOT (a) and SGPT (b) levels of each group. Note: $a$ - comparison with NC, $\beta$ -

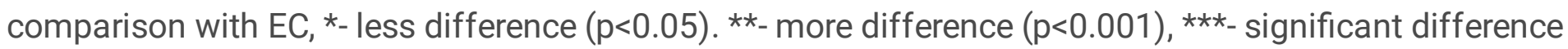
$(\mathrm{p}<0.0001)$ 

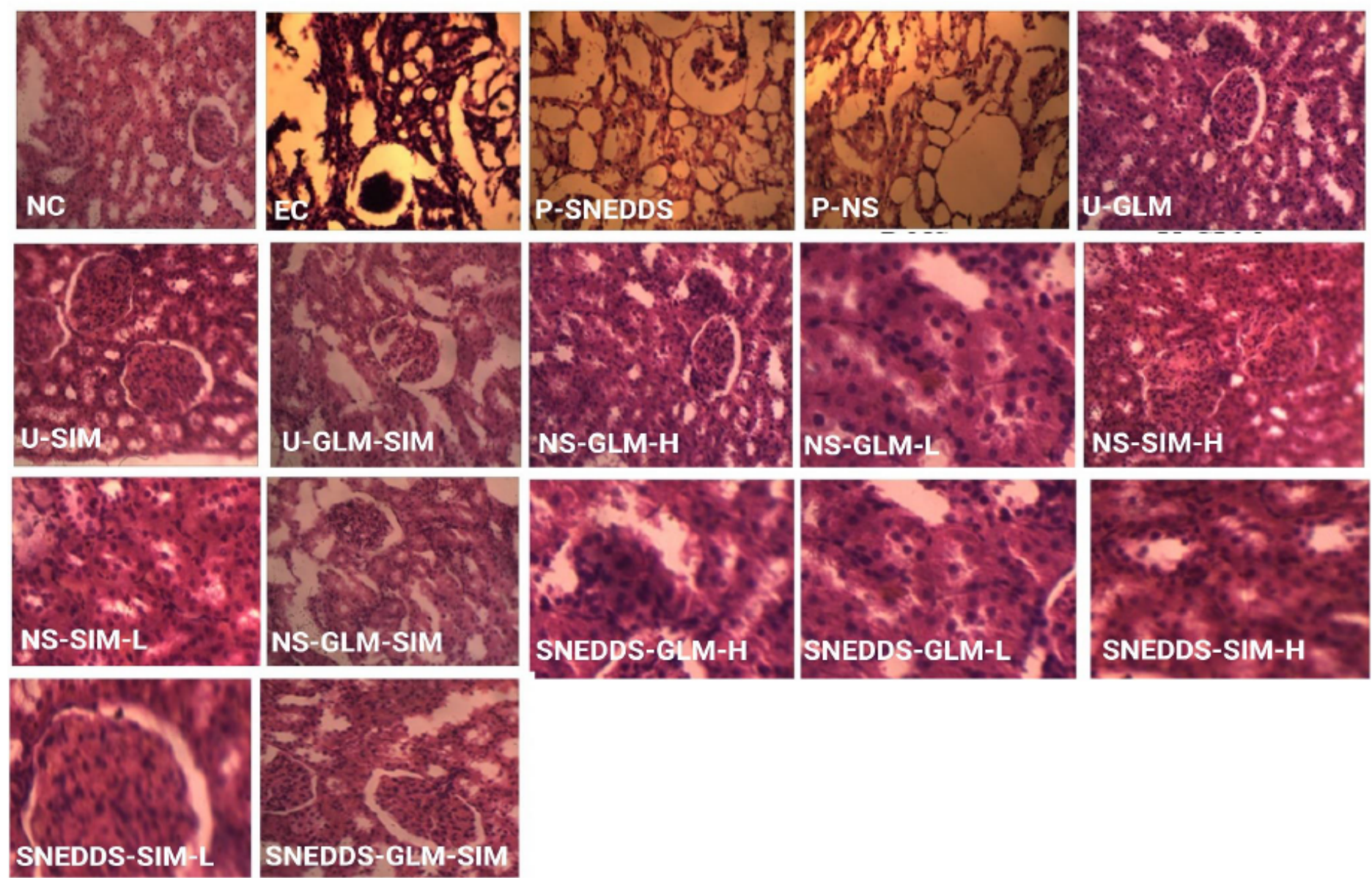

Figure 7

Histological sections of kidney 

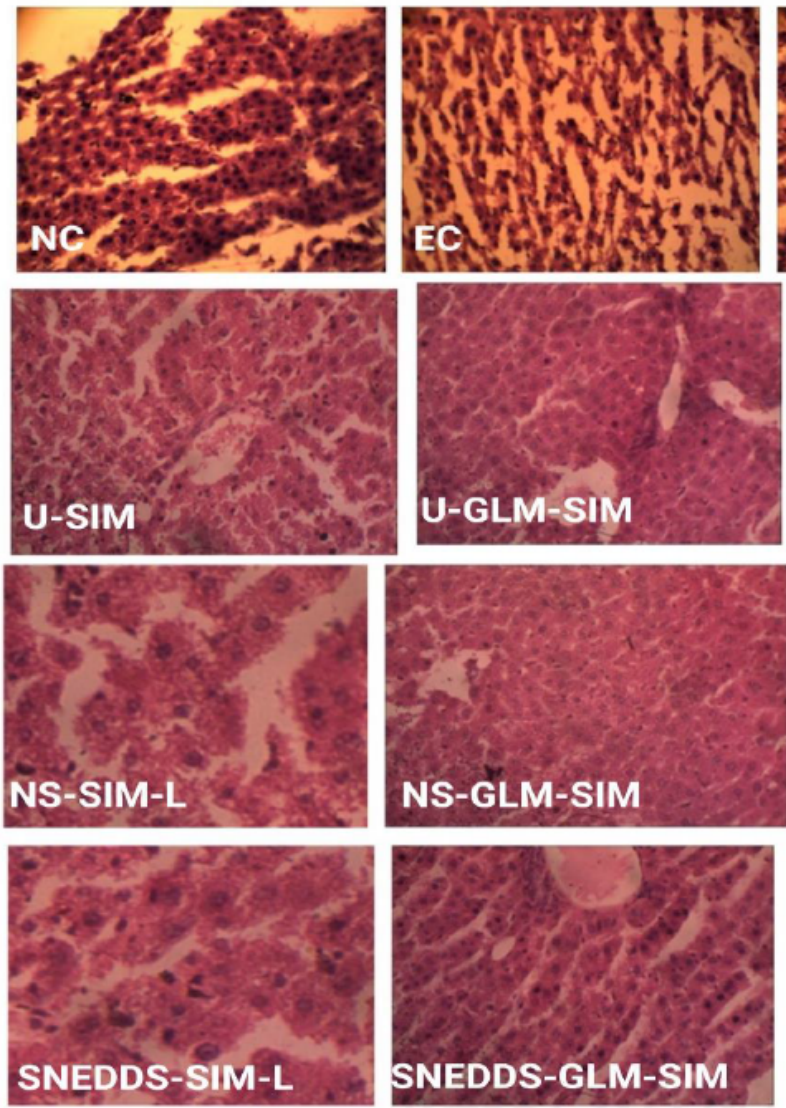
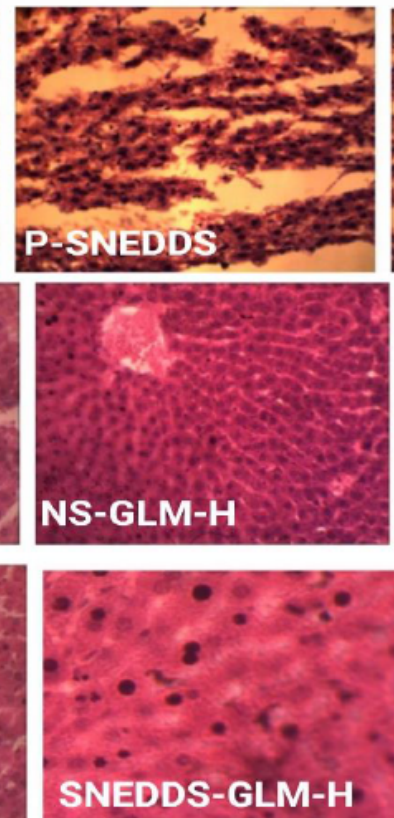

SNEDDS-GLM-H
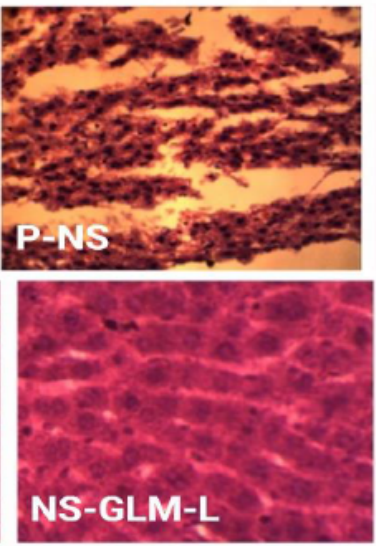

U-GLM
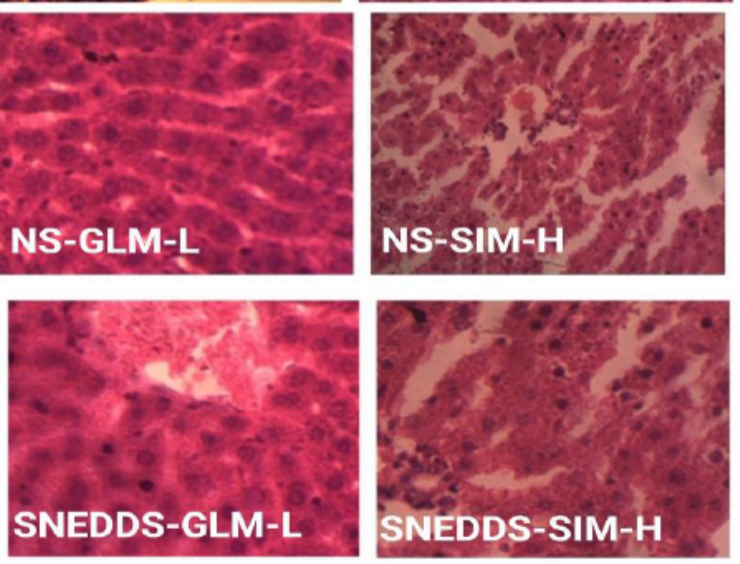

\section{Figure 8}

Histological sections of Liver 